Review Article

\title{
Functional, Cellular, and Molecular Remodeling of the Heart under Influence of Oxidative Cigarette Tobacco Smoke
}

\author{
Abdullah Kaplan, ${ }^{1}$ Emna Abidi, ${ }^{1}$ Rana Ghali, ${ }^{1}$ George W. Booz, ${ }^{2}$ Firas Kobeissy, ${ }^{3}$ and \\ Fouad A. Zouein ${ }^{1}$ \\ ${ }^{1}$ Department of Pharmacology and Toxicology, American University of Beirut Faculty of Medicine, Beirut, Lebanon \\ ${ }^{2}$ Department of Pharmacology and Toxicology, University of Mississippi Medical Center School of Medicine, Jackson, MS, USA \\ ${ }^{3}$ Department of Biochemistry and Molecular Genetics, American University of Beirut Faculty of Medicine, Beirut, Lebanon
}

Correspondence should be addressed to Firas Kobeissy; fk02@aub.edu.lb and Fouad A. Zouein; fz15@aub.edu.lb

Received 30 March 2017; Accepted 1 June 2017; Published 20 July 2017

Academic Editor: Victor M. Victor

Copyright (c) 2017 Abdullah Kaplan et al. This is an open access article distributed under the Creative Commons Attribution License, which permits unrestricted use, distribution, and reproduction in any medium, provided the original work is properly cited.

\begin{abstract}
Passive and active chronic cigarette smoking (CS) remains an international epidemic and a key risk factor for cardiovascular disease (CVD) development. CS-induced cardiac damage is divided into two major and interchangeable mechanisms: (1) direct adverse effects on the myocardium causing smoking cardiomyopathy and (2) indirect effects on the myocardium by fueling comorbidities such as atherosclerotic syndromes and hypertension that eventually damage and remodel the heart. To date, our understanding of cardiac remodeling following acute and chronic smoking exposure is not well elucidated. This manuscript presents for the first time the RIMD (oxidative stress (R), inflammation (I), metabolic impairment (M), and cell death (D)) detrimental cycle concept as a major player in CS-induced CVD risks and direct cardiac injury. Breakthroughs and latest findings in the field with respect to structural, functional, cellular, and molecular cardiac remodeling following chronic smoking exposure are summarized. This review also touches the genetics/epigenetics of smoking as well as the smoker's paradox and highlights the most currently prominent pharmacological venues to mitigate CS-induced adverse cardiac remodeling.
\end{abstract}

\section{Introduction}

Cardiovascular diseases (CVDs) remain the leading cause of morbidity and mortality worldwide. The World Health Organization (WHO) estimates a total of 17.5 million annual CVD deaths (31\% of total death) mostly due to myocardial infarction (MI) and strokes (http://www.who .int/cardiovascular_diseases/en/). Tobacco smoke is a major risk factor for CVDs, and chronic tobacco smoking (CS) increases the risk of coronary artery disease (CAD) by two- to fourfold $[1,2]$. WHO estimates a total of 5 million deaths yearly due directly to tobacco smoke and more than 600,000 deaths due to second-hand smoke. People who are exposed to second-hand smoke are at a $25-30 \%$ risk of developing heart disease and $20-30 \%$ risk of stroke [1]. Tobacco smoke contains more than 4720 compounds including well-known harmful chemicals such as polycyclic aromatic hydrocarbons, free radicals, and oxidative gases $[3,4]$. Although multiple studies show that oxidative compounds are primarily responsible of smoking-mediated myocardial injury $[3,5-9]$, the exact mechanisms of numerous potentially harmful smoke compounds remain poorly understood and require further investigation. This review highlights the effect of CS on structural, functional, and molecular cardiac remodeling based on most recent breakthroughs and latest publications. Promising pharmacological interventions that protect the heart from the cigarette smoking epidemic are summarized in this manuscript.

\section{Cardiac Remodeling Post-CS: Structural and Functional Level}

Cardiac remodeling occurs in response to both physiological and pathological stimuli including exercise, myocardial 
infarction, arterial hypertension, valvular diseases, and myocarditis. In pathological response, both molecular and cellular changes can lead to either ventricular hypertrophy and/or dilatation that are functionally translated into diastolic and/or systolic dysfunction. The term smoke cardiomyopathy was first introduced by Gvozdjakova et al. to refer to metabolic and morphological alterations in the rabbit myocardium after chronic CS and in the absence of comorbidities $[10,11]$. Numerous studies highlight the negative impact of acute and chronic smoking on ventricular systolic and diastolic function in human and rodents [12-18]. Direct and indirect toxic effects to the myocardium have been reported with CS exposure both clinically and experimentally [19]. Direct effects such as myocardial ischemia, necrosis and fibrosis, focal myocardial hemorrhage, focal myocarditis, myocardial fiber swelling, functional and structural alteration of myocardial mitochondria, coronary vasocontraction, and cardiac arrhythmias have been documented [19]. CS indirect effects on the heart are attributed to blood pressure increase, plasma cholesterol level alteration, increased plasma viscosity and platelet aggregations, T-cell function alteration, and increased inflammatory profile [19]. CS-mediated increase in blood pressure and heart rate was attributed to sympathetic outflow enhancement to blood vessels and heart, respectively [20]. However CS-induced sympathetic effects on the heart did not increase cardiac inotrope mainly due to CS-mediated myocardial oxygen deficiency due to carbon monoxide and carboxy hemoglobin accumulation [21]. Cardiac hemodynamic changes in rodents exposed to smoke with different exposure times are documented. Mice exposed to 32 weeks of smoke showed impaired systolic and diastolic function when left ventricular (LV) pressure-volume relationship was assessed at high afterloads [22]. Dawley rats exposed to 5 weeks of smoke exhibited a significant increase in LVEDD and LVESD along with a significant decrease in FS when compared to the control group [23]. In addition to systolic dysfunction and increased left ventricular systolic volume, rats exposed to 8 weeks of CS experienced an enlargement of the left atrium [24]. Table 1 summarizes a selective list of preclinical CS studies and their impact on the structure and function of the heart in the presence or the absence of comorbidities such as MI or volume overload.

\section{Cardiac Remodeling Post-CS: Cellular and Molecular Level}

Myocardium cellular and molecular impairment following CS is directly related to at least 4 interchangeable mechanisms that are termed RIMD in this review and include oxidative stress (R), inflammation (I), metabolic impairment $(\mathrm{M})$, and cell death (D) $[3,9,11,25,26]$. In response, matrix metalloproteinase (MMP) activation, mitogen-activated protein kinase (MAPK) activation, mitochondrial dysfunction, lipotoxicity, neurohormonal imbalance, gap junction remodeling, immune cell infiltration, and other events contribute to the adverse remodeling of the myocardium [3, 9, 23, 24, 2730]. Direct adverse effects of CS on myocardium are exacerbated by their general effects on the cardiovascular system, creating a vicious adverse remodeling cycle that can acutely or progressively damage the heart (Figure 1). In fact, CS is directly responsible for several clinical atherosclerotic syndromes, including stable and unstable angina, aortic atherosclerosis, coronary lesion, and sudden death [31-34]. A large number of studies support the direct stimulatory effect of CS on multiple components of atherosclerotic syndrome progression including, endothelial/vasomotor dysfunction [35-42], dyslipidemia [43-46], inflammation [47-50], platelet dysfunction [51-53], and alteration in antithrombotic, prothrombotic, and fibrinolysis factors [54-57].

3.1. Oxidative Stress. Oxidative stress occurs as an imbalance between reactive oxygen species (ROS) production and antioxidant defense mechanisms within the cell. Under physiologic conditions, ROS production occurs as result of oxygen metabolism and plays a vital role in cell signaling $[58,59]$. However, unbalanced and excessive ROS production in response to exogenous and endogenous stimuli can cause lipid peroxidation, DNA strand breaks, and other forms of intracellular injury which are detrimental to cellular structure and function [58]. In addition to intracellular ROS induction, cigarette smoke contains substantial amounts of ROS and other chemicals that diminish the intracellular antioxidant mechanisms [5, 9]. As a result, inflammation, MMP activation, cardiac fibroblast proliferation, and intracellular remodeling pathway stimulation contribute to CS-induced cardiac remodeling $[9,60]$. Talukder et al. showed that both mononuclear and polymorphonuclear blood cells from 32 week CS-exposed mice generated more $\mathrm{H}_{2} \mathrm{O}_{2}$ and superoxide than controls leading to systemic oxidative stress and mild cardiac hypertrophy in mice that were not otherwise predisposed to disease [22]. Santos et al. reported similar pathology in rats exposed to 8 weeks of CS. Their analysis revealed direct cardiotoxicity characterized by decreased glutathione peroxidase and superoxide dismutase activity, decreased fatty acid (FA) oxidation, and subsequent increase in ROS formation, lipotoxicity (increased lipid peroxidation), and mitochondrial dysfunction [24]. Duarte et al. assessed the impact of CS on ventricular remodeling following myocardial infarction. CS exacerbated LV remodeling post-MI with a significant increase in glutathione disulfide (GSSG) and a decreased glutathione (GSH), GSH/GSSG ratio in both the heart and the liver [25]. Those findings suggest an impaired systemic antioxidant defense and further support the systemic oxidative impact of CS on cardiac remodeling. Multiple studies support the systemic oxidation impact using drugs with antioxidant properties to rescue CS-induced cardiac remodeling. Those rescue-type therapies are discussed in the pharmacological venue section of this review.

3.2. Inflammation. Regardless of the underlying mechanisms, cardiac remodeling is often accompanied by an inflammatory response. Several clinical and preclinical studies positively correlate heart failure with high levels of proinflammatory cytokines [61-66], and an inflammatory response post-CS exposure has been well documented. In the case of smoking, combustion can trigger the production of ROS that are normally not present in the leaf or the ash [67] but are enriched in both the gaseous and particulate 


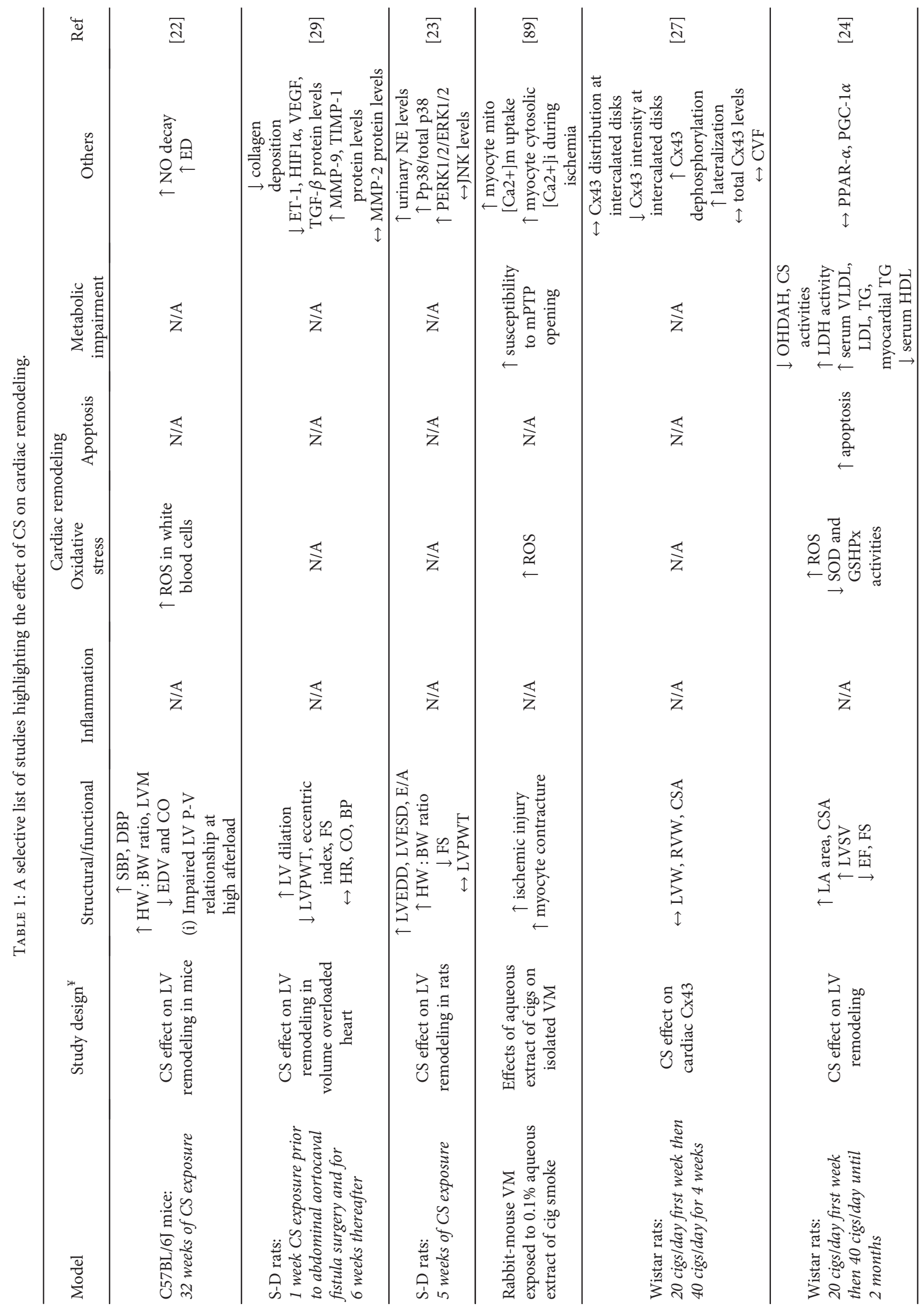




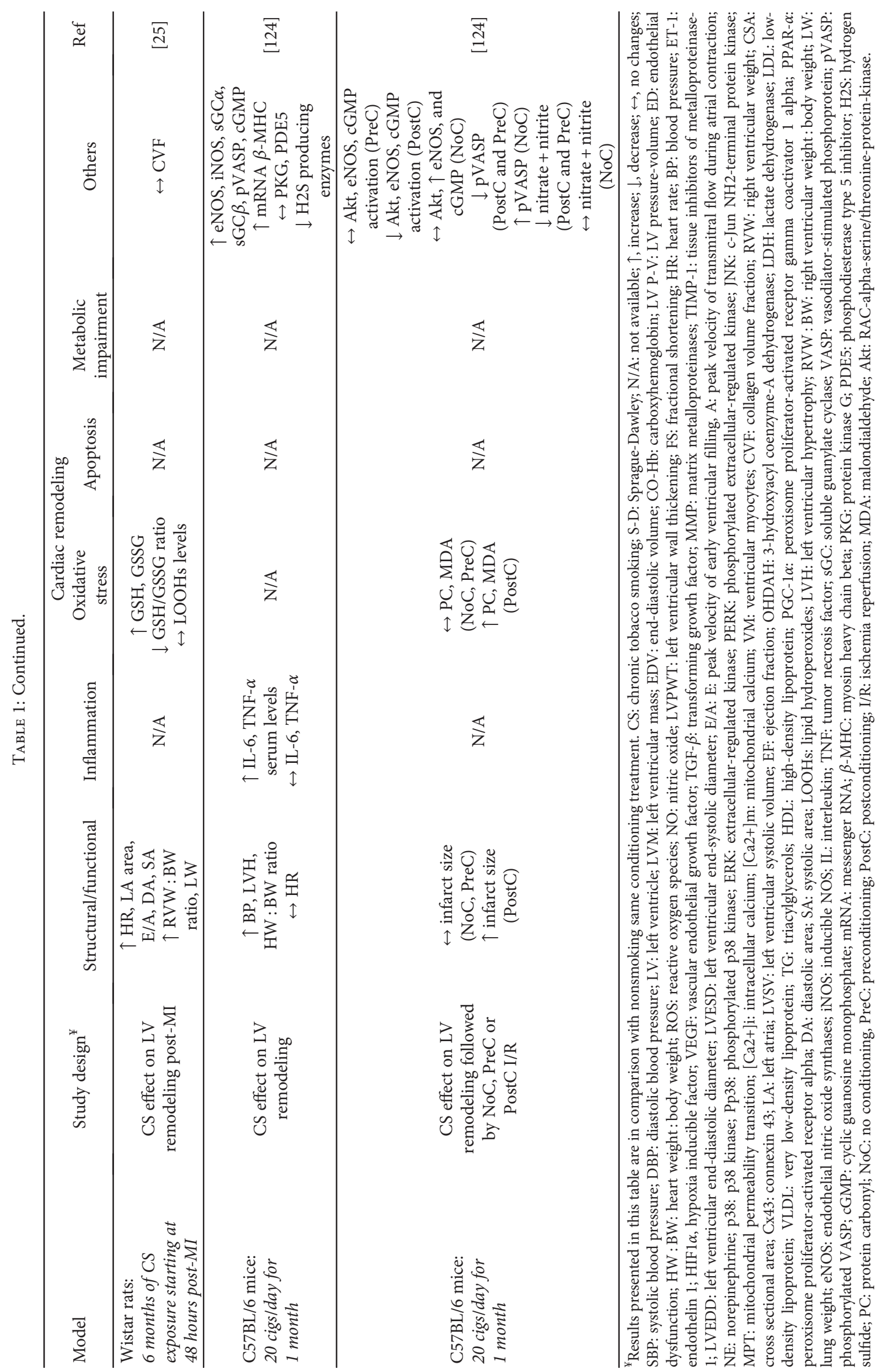




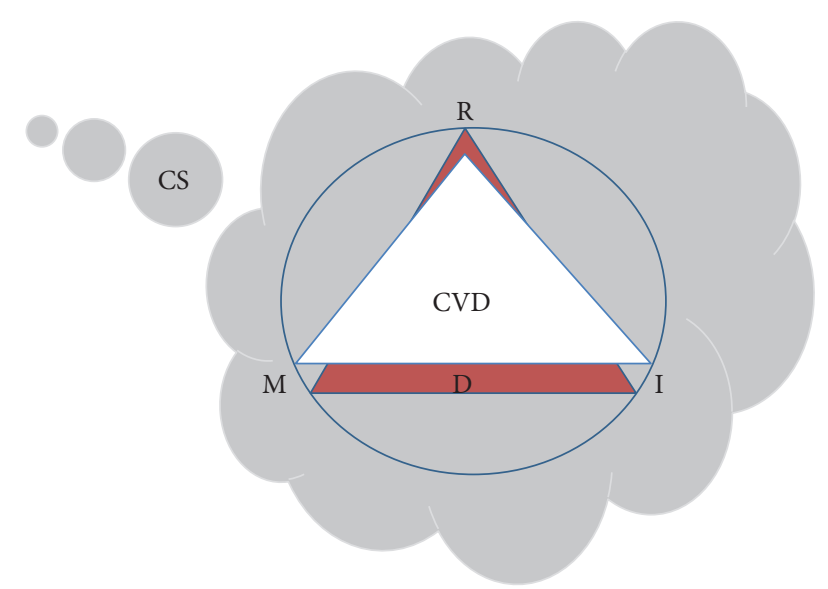

FIgURE 1: Vicious and detrimental cycle of CS-induced cardiovascular injury. In addition to exogenous ROS delivery, chronic smoking exposure attenuates antioxidant defenses and increases endogenous ROS formation. Oxidative stress (R) will eventually trigger an inflammatory response (I) and metabolic impairment (M) and subsequent cell death (D) that fuel ROS formation. This chronic phenomenon could lead to CVD. The presence of CVD exacerbates CS-mediated RMID process detrimental effects.

components of smoking $[68,69]$. Such alteration in ROS production due to cigarette smoking has drastic effect on the host immunity affecting the innate immunity in the airway mucosa, as well as adaptive immunity at the systemic level $[70,71]$. ROS play a crucial role in the inflammation process by initiating a variety of ROS-sensitive signaling pathways including mitogen-activated protein kinases (MAPKs) and a number of transcription factors, such as nuclear factor $-\kappa \mathrm{B}$ $(\mathrm{NF}-\kappa \mathrm{B})$. Subsequently, inflammatory gene expression is triggered increasing the production of proinflammatory cytokines such as interleukin-8 (IL-8) and tumor necrosis factor-alpha (TNF- $\alpha$ ) $[70,72]$. The secretion of these inflammatory cytokines leads to chronic immune cell recruitment and inflammation [73]. Walters and colleagues demonstrated for the first time that smoke stimulates IL-8 release from monocytes and macrophages in an oxidant-mediated phenomenon and mainly by acting via activator protein-1 (AP-1) pathway along with tonic levels of NF- $\kappa \mathrm{B}$ activation [73]. Walters work also revealed that inhibition of IL-8 release is steroid resistant only when stimulation is primed with IL- $1 \beta$. Those findings could elucidate the mechanisms behind clinical, smoking-induced, inflammatory response resistance to glucocorticoid treatment [74]. Although oxidative stress and inflammation are normally coassociated together, Das et al. demonstrated that inflammatory response follows oxidative stress in a two-week margin following CS in pigs [9]. Oxidative damage was detected after two weeks of CS exposure while proinflammatory cytokines including TNF- $\alpha$ and IL- $1 \beta$ were detected after 4 to 6 weeks of CS. Neutrophil infiltration into the myocardium was observed around 6 to 8 weeks following CS [9]. These studies highlight the effect of oxidative damage in releasing danger associated molecular patterns (DAMPs) that trigger an immune cell response and subsequent inflammation
[75]. The inflammatory profile was assessed by Zhou et al. in two independent studies following 4 months of CS exposure in rats. Their findings revealed an enhanced cardiac gene expression of proinflammatory cytokines including IL- $1 \beta$, IL-6, and TNF- $\alpha$ and serum circulating levels of Hs-CRP, IL-6, TNF- $\alpha$, IL- $1 \beta$, and MCP $[60,76]$ suggesting systemic inflammation. Other studies supported both the upregulation of other proinflammatory cytokines including IL-2, IL-8, IFN- $\gamma$, and GM-CSF and the downregulation of anti-inflammatory cytokines such as IL-10 [77, 78]. However, other studies reported controversial results. Duarte et al. and Santos et al. observed that CS did not impact myocardial levels of multiple cytokines inducing IFN- $\gamma$, TNF- $\alpha$, IL-10, and ICAM-1 $[28,79]$. One explanation for these contradicting findings could be the inconsistencies between studies as far as CS exposure time and concentration. Table 1 highlights different findings with respect to CS exposure protocols.

3.3. Metabolic Impairment. Cellular metabolic impairment constitutes another source of oxidative stress that fuels oxidative damage and subsequent inflammatory response post-CS. Low ROS levels are normally generated during oxidative phosphorylation in mitochondria [80] and kept within a normal range due to the presence of antioxidant mechanisms like glutathione, vitamin E, catalases, peroxidases, and superoxide dismutase (SOD) [81]. Under normal conditions, oxidants regulate intracellular processes, but excess oxidants induce irreversible damage to cellular components leading to altered cellular functions or apoptosis [82]. In vitro, ROS were shown to cause mitochondrial injury by attenuating mtDNA-encoded mRNA transcripts, altering mitochondrial protein synthesis and decreasing cellular ATP levels and mitochondrial redox potential [83]. By virtue of their importance in cell signaling and apoptosis, mitochondria may modify cellular energy production and regulatory pathways in cardiovascular cells [84]. Mitochondrial signaling disruption due to chronic oxidant exposure could thereby lead to reduced energy production and induce cell death mainly in tissues with low energetic thresholds [84]. Morphological and functional changes in myocardial mitochondria including swelling, external membrane alteration, lipid accumulation, oxidative phosphorylation rate reduction, ROS production, and mitochondrial permeability transition pore (mPTP) opening have all been reported in animals following CS [11, 26, 85-87]. Gvozdjakova et al. linked the "smoke cardiomyopathy" mainly to mitochondrial dysfunction $[11,26]$. They showed a significant decrease in respiration as well as in the phosphorylation rate of mitochondria, diminished cytochrome oxidase activity, and decreased coenzyme Q10 (CoQ10) levels, three weeks following passive CS exposure in rabbits. CoQ10 is a critical component of normal mitochondrial ETC function and plays an important role in reducing lipid peroxidation and ROS scavenging [88]. Santos et al. reported, in their 2-month CS exposure study in rats, abnormalities in energy metabolism, including lipotoxicity and oxidative stress [24]. This study revealed impaired mitochondrial respiration through enhanced lactate dehydrogenase activity and decreased citrate synthase activity, and an 


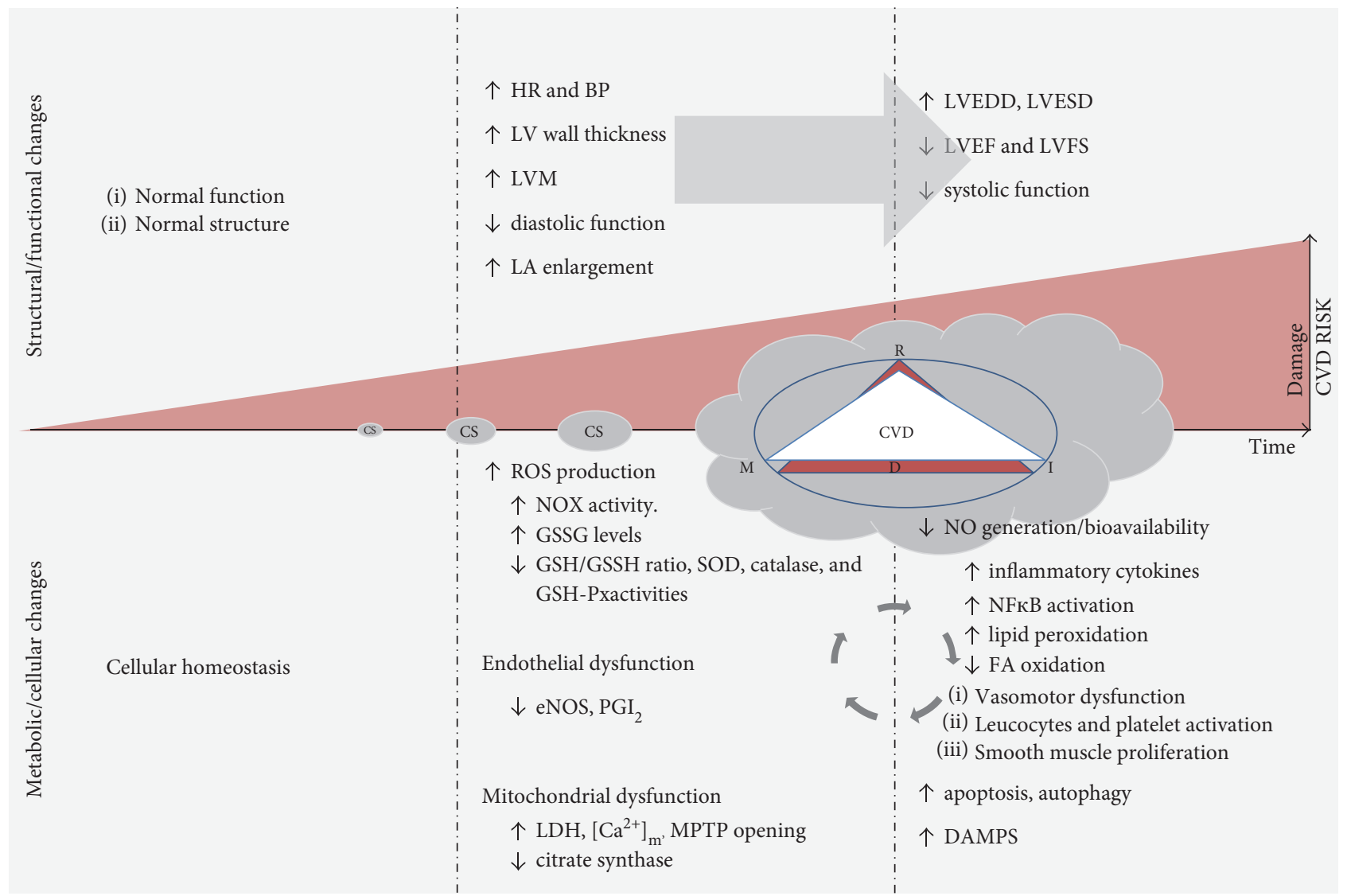

FIGURE 2: The impact of chronic smoking exposure on CVD risk and cardiac structural, functional, and cellular damage. CS-based RMID cause metabolic and cellular damages that alter cardiac structure and function and increase CVD risk and myocardial damage. HR, heart rate; $\mathrm{BP}$, blood pressure; LV, left ventricle; LVM, left ventricular mass; LA, left atria; LVEDD, left ventricular end-diastolic diameter; LVESD, left ventricular end-systolic diameter; LVEDV, left ventricular end-diastolic volume; LVEF, left ventricular ejection fraction; LVFS, left ventricular fractional shortening; ROS, reactive oxygen species; NOX, NADPH oxidase; GSSG, glutathione disulfide; GSH/ GSSH, glutathione disulfide/glutathione ratio; SOD, superoxide dismutase; GSH-px, glutathione peroxidase; eNOS, endothelial nitric oxide synthases; $\mathrm{PGI}_{2}$, prostacyclin; $\mathrm{LDH}$, lactate dehydrogenase; $\left[\mathrm{Ca}^{2+}\right]_{\mathrm{m}}$, mitochondrial calcium; MPT, mitochondrial permeability transition; NF- $\kappa$, nuclear factor- $\kappa \mathrm{B}$; FA, fatty acid; DAMPs, danger-associated molecular patterns.

impaired FA oxidation as evidenced by reduction in 3hydroxyacyl coenzyme-A dehydrogenase level and increased triglyceride density in cardiac tissue [24]. Last but not least, Yamada et al. concluded that clinically relevant concentration of aqueous extract of cigarette smoke increases cardiomyocyte mitochondrial $\mathrm{Ca}^{2+}$ load during simulated ischemia and the susceptibility of mPTP opening and subsequent cell death. These findings were partly attributed to tobacco smoke-induced ROS [89]. In summary, metabolic impairment post-CS exposure plays a major role in cellular dysfunction by participating in the vicious cycle of CSinduced RIMD (Figure 2).

3.4. Cell Death. ROS, inflammation, and metabolic impairment post-CS exposure predispose cardiac cells to death $[9,24,30,60,90]$. In their vitamin C-deficient guinea pig model, Das et al. documented the induction of both intrinsic (cytochrome $\mathrm{C}$ release, p53 phosphorylation, increased $\mathrm{Bax} / \mathrm{Bcl}-2$ ratio, and activation of caspase 3 ) and extrinsic (TNF- $\alpha$ upregulation and caspase 8 activation) pathways of apoptosis in the myocardium in a time-dependent manner after CS exposure [9]. High cardiomyocyte apoptotic rate was also shown via annexin V/PI staining by Zhou et al. in a CS rat model [60]. On a separate study, Zhou et al. reported the induction of JNK and P38 of MAPK signaling pathways and the inhibition of PI3K/ AKT pathways in the myocardium following CS exposure to rats [30]. Both pathways are known to be involved in cellular apoptosis [91, 92]. Increased extracellular signalregulated kinase $(\mathrm{ERK} 1 / 2)$ and $\mathrm{p} 38$ of MAPK were also associated with CS-induced left ventricular remodeling [23]. In addition to apoptosis, autophagy was also reported to play a pathogenic role in smoking-induced left ventricular systolic dysfunction [30,93].

3.5. Genetic and Epigenetics of Smoking-Induced CVD. Genetic and epigenetic predisposition to smoking-induced CVD have been established but barely elucidated. One renowned genetic example is the interaction of apolipoprotein E (ApoE) variants with smoking. ApoE isoform, $\epsilon 4$, is associated with increased oxidized lipoprotein production and subsequent CVD risks in the presence of smoking- 
induced excessive $\operatorname{ROS}[94,95]$. Sequence variants in multiple other proteins including TGF- $\beta$, lipoprotein lipase, IL-18, IL-6, and prothrombin have been involved in smoking-related CVD risks [94, 96-98]. In addition to genetic variation, epigenetics of CVD gained significant attention in recent years. In the context of tobacco-smoking, few epigenetic patterns associated with CVD have been recognized [99, 100]. Recent epigenome wide studies identified a significant link between coagulation factor II receptor-like 3 (F2RL3) lower methylation and smoking behavior [99, 101]. F2RL3 gene encodes for protease-activated receptor 4 (PAR4), a protein that highly correlates with multiple cardiovascular pathophysiological events including inflammation, platelet function, myocardial injury, and death [101-104]. Other studies highlighted the association of smoking with alteration of microRNA expression in spermatozoa and subsequent potential adverse outcomes in offspring [105]. Evidence of smoking interaction with a flavin-containing monooxygenase 4 (FMO4) variant has also been reported with respect to coronary heart disease (CHD) [106]. Identifying genetic and epigenetic association with smoking-induced CVD is a promising tool to improve cardiovascular risk prediction, personalized prevention, and intervention in smokers. However, to date, gene-smoking interaction and pattern discoveries remain highly novel, multifactorial, and technically and statistically very challenging.

\section{The Smoker's Paradox}

Although history of smoking highly correlates with adverse cardiovascular risk, an old-new phenomenon known as "smoker's paradox" emerged, describing the potential beneficial effects of smoking on the cardiovascular system. The smoker's paradox was first noted in 1977 on a group of acute MI patients and later confirmed in multiple clinical cardiovascular studies including fibrinolysis, acute stroke, cardiac arrest, and ST elevated MI (STEMI) [107-111]. Multiple preclinical studies confirmed the paradoxical effect of smoke on mortality after MI and attributed the beneficial effects to cardiac gap junction remodeling and preconditioning $[27,112]$. Smoking was independently associated with improved survival in patients with cardiac arrest and lower inpatient mortality in acute ischemic stroke and STEMI [107, 110, 111]. Results were attributed in part to changes in vascular reactivity due to a global smoking-induced ischemic conditioning. On average, compared with nonsmokers, smokers are consistently younger on hospital admission with fewer comorbidities and better overall prognosis. Nonetheless, GUSTO-1 trial revealed a $25 \%$ lower mortality rate in smokers 30 days postAMI thrombolysis, even after adjusting for age, sex, blood pressure, and other covariates [108]. In contrast, recent SYNTAX trial findings revealed that smoking was associated with a higher rate of death/MI/stroke (86\% MI hazard) at 5 years follow-up following coronary artery bypass grafting (CABG) and percutaneous coronary intervention (PCI) for stable CAD-treated smoker patients [113]. SYNTAX recommended smoke cessation to improve revascularization benefits. The contradictory findings between GUSTO-1 and SYNTAX could be explained as follows: (1) the GUSTO-1 trial studied the impact of short-term (30 days) impact of smoke versus 6 months to 5 years for SYNTAX; (2) the GUSTO-1 trial focused on thrombolysis revascularization following AMI versus CABG and PCI in stable CAD for SYNTAX; and (3) SYNTAX allowed smokers who quit during trial to be analyzed as nonsmokers during the interval in which they quit $[108,113]$. Multiple recent clinical studies denied CS protective effects and concluded that smoker's paradox is in fact a pseudoparadox [114-116]. Their findings revealed no relevance of smoking paradox among patients undergoing primary PCI [114]. Additionally, smoking history had no significant effects on infarct size 30 days postMI [115]. In fact, a growing consensus support the hypothesis that smoker's paradox is largely attributed to differences in demographics and clinical baseline risks and that smoking survival benefit in univariable analysis is mostly related to younger population with low CV risks, shorter time exposure, and absence of comorbidities [116].

Clearly the long-known clinical controversy behind the paradox of smoking is shrinking. However, experimental evidence of CS-induced cardioprotection could be related to potential unknown bioactive protective cigarette compounds. Their discovery and isolation from harmful compounds could devise novel therapeutic strategies to improve the overall prognosis following acute cardiac events.

\section{Pharmacological Venues}

Smoking cessation is currently the most adapted strategy to treat smoking habit. A variety of smoking cessation therapies are clinically available and divided into two major groups: drug-based therapy and drug-free therapy [117]. Drugbased therapy involves nicotine replacement therapy (NRT) including nicotine gum and nicotine skin patches and prescription drug therapy (PDT) including nicotine receptor antagonists (bupropion hydrochloride) and nicotine receptor partial agonist (varenicline) [117, 118]. Drug-free therapy involves awareness and counseling to reduce smoking progressively and rapidly $[119,120]$. However, smoking cessation compliance among smokers is limited due to drug side effects and/or socioeconomic constraints which increase the chances of relapse $[121,122]$. In CVD, a novel therapeutic strategy, as adjunct to smoking cessation attempts, is to clinically focus on reducing the smoking adverse effects on the heart in both acute and chronic events such as smoking cardiomyopathy or AMI to improve the overall prognosis. Multiple preclinical studies have successfully documented the importance of multiple supplements and drugs in reducing the adverse effect of smoking on the heart (Table 2). For instance, Rafacho et al. revealed that vitamin D supplementation attenuates cardiac remodeling post-CS in rats by increasing superoxide dismutase and catalase activity and lowering lipid oxidation [123]. Das et al. reported that vitamin C supplementation to CS-exposed guinea pig prevented adverse cardiac remodeling post-MI mainly by reducing and inactivating $\mathrm{p}$-benzoquinone ( $\mathrm{p}-\mathrm{BQ})$, a redox cycling agent produced from $\mathrm{p}-\mathrm{BSQ}$ of CS and a major factor that produces ROS, oxidative damage, and apoptosis $[9,123]$. Reduced oxidative stress and improved cardiac bioenergetics were 


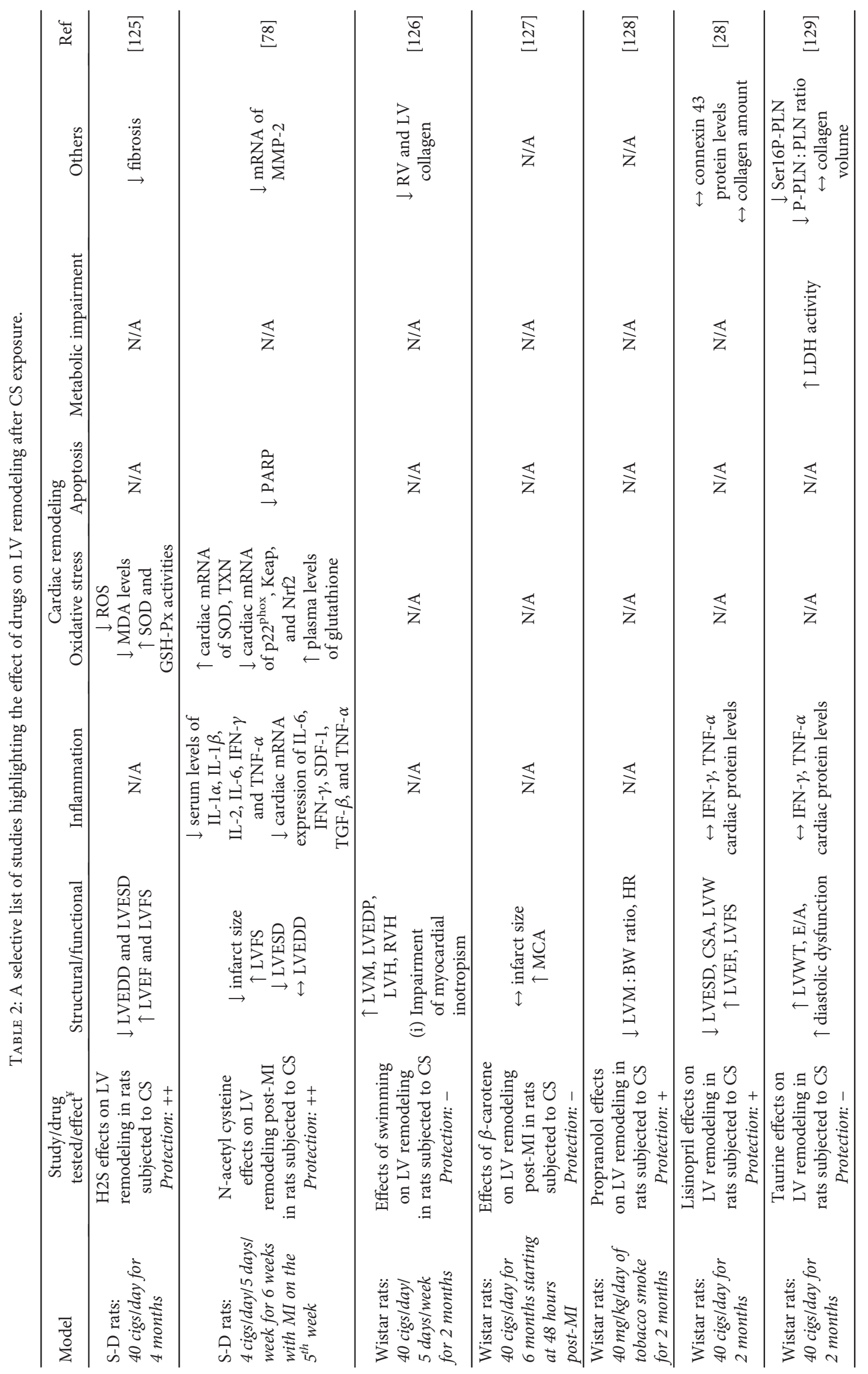




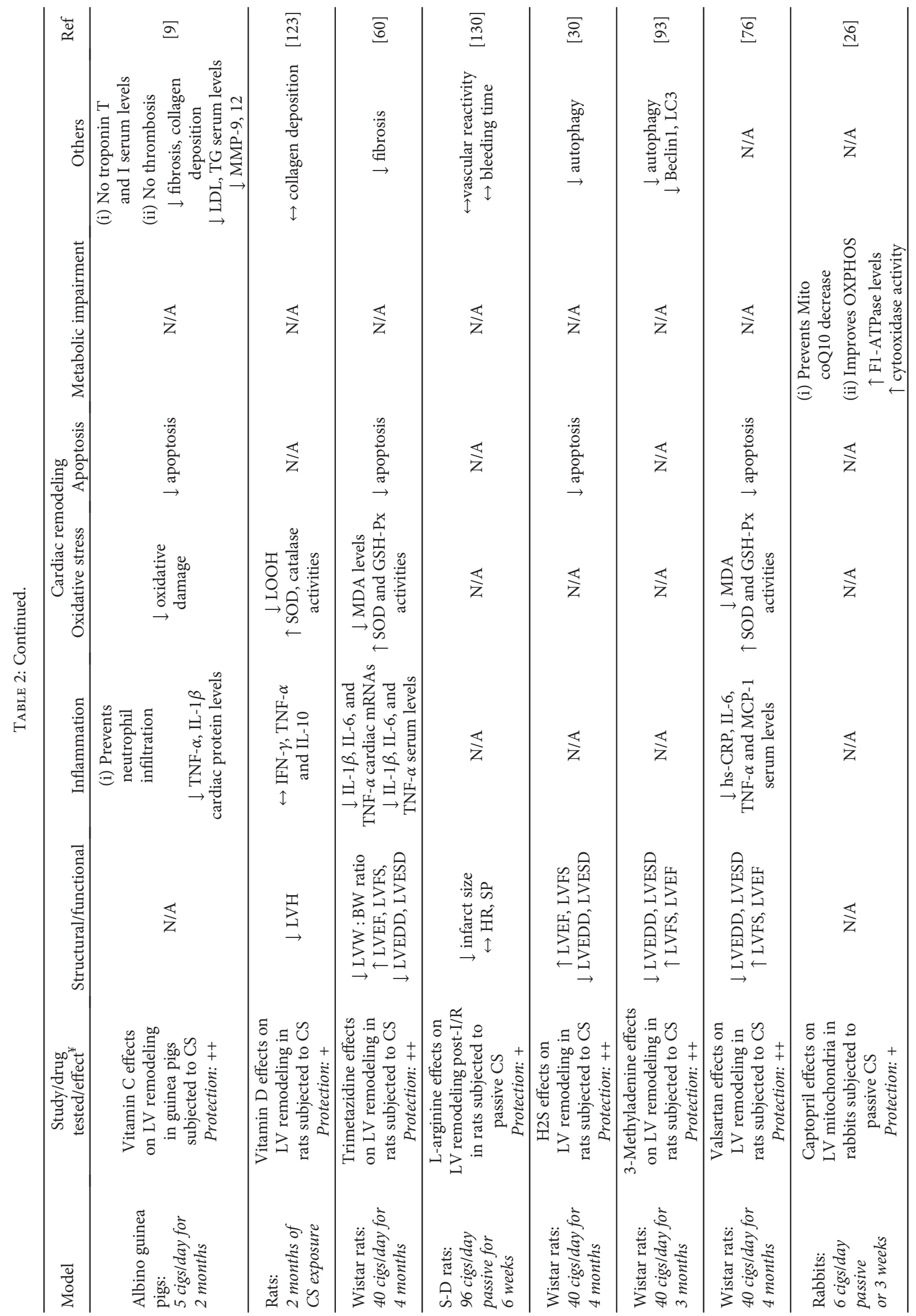




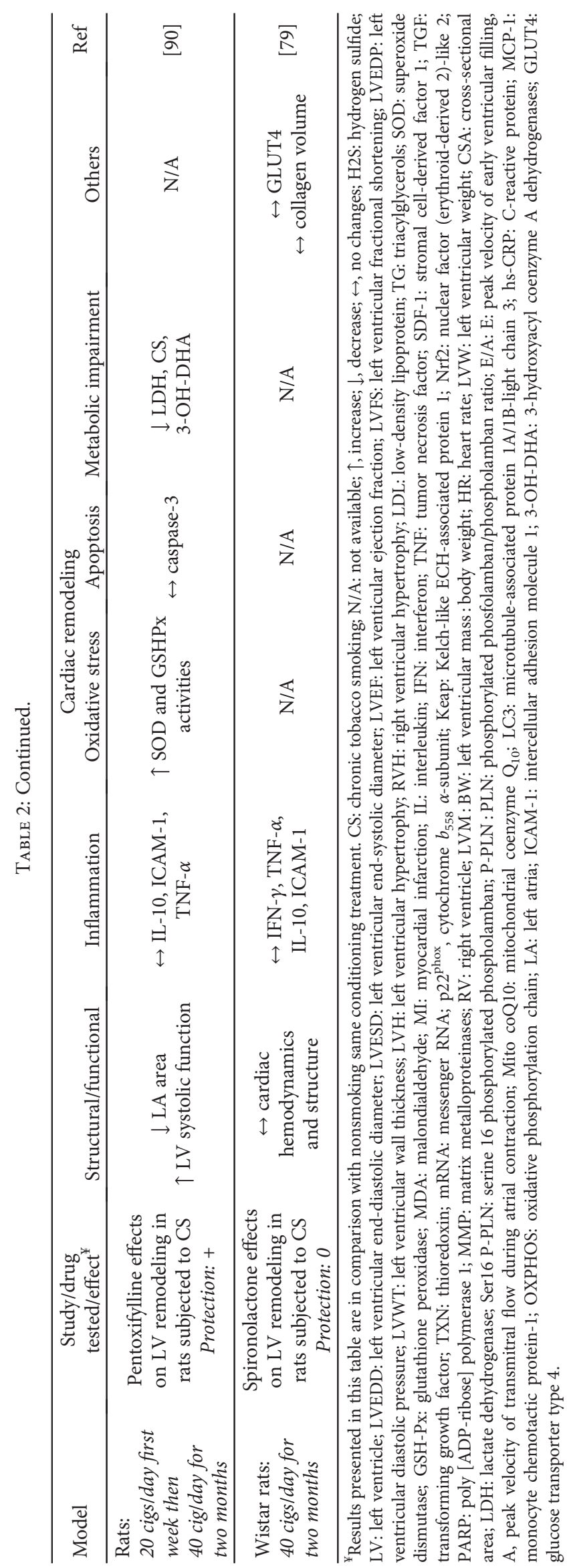


reported in pentoxifylline (nonselective phosphodiesterase (PDE) inhibitor)-treated rats that were exposed to CS [90]. In addition to ROS scavenging, attenuating inflammatory and apoptotic response post-CS exposure has also been reported [30, 60, 76, 78]. Multiple drugs including trimetazidine, N-acetyl cysteine, hydrogen sulfide (H2S), and valsartan reduced smoking-induced inflammation and/or apoptosis and, subsequently, modulated smoking-mediated left ventricular dysfunction $[30,60,76,78]$. In conclusion, drugs that target the detrimental smoking-induced cycle of RIMD seem to exert beneficial effects by attenuating CSinduced cardiac dysfunction in the presence or absence of acute cardiac events. Of note, RIMD branches are dependent and interchangeable, meaning that any drug that affects one branch, such as ROS, could affect the concept as a whole. Those approaches should be further explored in the clinics with the attempt to increase the overall prognosis of chronic smokers by either preventing smoking-induced cardiomyopathy or improving cardiac remodeling following an acute cardiac injury such as MI.

\section{Conclusions and Future Perspectives}

Despite increased social awareness, marketing restraints, tobacco taxation, and available smoking cessation rehabs, active and passive smoking remains a worldwide challenging epidemic with high CVD risks and other fatal diseases. In this review, we presented for the first time the concept of RIMD to explain CS-mediated adverse effects on the myocardium. CS impact on the myocardium is multifactorial and directly related to 4 main interchangeable mechanisms including ROS generation, metabolic impairment, inflammation, and cell death. Chronic exposure to smoking induces molecular and cellular alteration that could translate into myocardial structural and functional changes that vary between individuals depending on their genetic and epigenetic predisposition. To date, no clinically effective therapy is adapted to reduce CS-induced adverse effects on the myocardium in the presence or absence of comorbidities. Alternative solutions to smoking cessation should be implemented. For instance, our current understanding of functional, structural, molecular, cellular, genetic, and epigenetics of smokingassociated cardiac remodeling should be escalated in order to devise novel therapeutic strategies that limit smokingmediated CVD and associated comorbidities. Multiple promising supplements and drugs remain in the preclinical phase with no obvious signs of potential clinical adoption.

\section{Abbreviations}

$\begin{array}{ll}\text { CVDs: } & \text { Cardiovascular diseases } \\ \text { IL: } & \text { Interleukin } \\ \text { LV: } & \text { Left ventricle } \\ \text { MI: } & \text { Myocardial Infarction } \\ \text { MMPs: } & \text { Matrix metalloproteinases } \\ \text { CS: } & \text { Chronic tobacco smoking } \\ \text { CAD: } & \text { Coronary artery disease }\end{array}$

LVEDD: Left ventricular end-diastolic diameter

LVESD: Left ventricular end-systolic diameter
FS: $\quad$ Fractional shortening

RIMD: Oxidative stress-inflammation-metabolic impairment-cell death

MMP: Matrix metalloproteinase

MAPK: Mitogen-activated protein kinase

ROS: $\quad$ Reactive oxygen species

GSH-px: Glutathione peroxidase

SOD: $\quad$ Superoxide dismutase

FA: $\quad$ Fatty acid

GSSG: Glutathione disulfide

GSH: Glutathione

MAPKs: Mitogen-activated protein kinases

NF- $\kappa$ B: $\quad$ Nuclear factor $-\kappa \mathrm{B}$

TNF- $\alpha$ : Tumor necrosis factor-alpha

coQ10: Coenzyme 10

ERK1/2: Extracellular signal-regulated kinase

CABG: Coronary artery bypass grafting

PCI: Percutaneous coronary intervention.

\section{Conflicts of Interest}

The authors confirm that there are no conflicts of interest.

\section{Acknowledgments}

This work was supported by grants from the American University of Beirut (Seed Grant no. 100410 and MPP Grant no. 320145) to Fouad A. Zouein. The authors are grateful for the generous support of the Department of Pharmacology and Toxicology of the University of Mississippi Medical Center (George W. Booz) and the Department of Pharmacology and Toxicology at the American University of Beirut. The authors also thank Dr. Jessica Saliba for her valuable comments and edits.

\section{References}

[1] The Health Consequences of Smoking-50 Years of Progress: A Report of the Surgeon General, Atlanta, GA, 2014.

[2] Centers for Disease, C, “The surgeon general's 1989 report on reducing the health consequences of smoking: 25 years of progress," MMWR Supplements, vol. 38, no. 2, pp. 1-32, 1989.

[3] B. P. Rafacho, P. S. Azevedo, B. F. Polegato et al., "Tobacco smoke induces ventricular remodeling associated with an increase in NADPH oxidase activity," Cellular Physiology and Biochemistry, vol. 27, no. 3-4, pp. 305-312, 2011.

[4] H. Yao, I. Edirisinghe, S. R. Yang et al., "Genetic ablation of NADPH oxidase enhances susceptibility to cigarette smoke-induced lung inflammation and emphysema in mice," The American Journal of Pathology, vol. 172, no. 5, pp. 1222-1237, 2008.

[5] J. A. Ambrose and R. S. Barua, "The pathophysiology of cigarette smoking and cardiovascular disease: an update," Journal of the American College of Cardiology, vol. 43, no. 10, pp. 1731-1737, 2004.

[6] S. S. Barbieri, E. Zacchi, P. Amadio et al., "Cytokines present in smokers' serum interact with smoke components to enhance endothelial dysfunction," Cardiovascular Research, vol. 90, no. 3, pp. 475-483, 2011. 
[7] D. Bernhard and X. L. Wang, "Smoking, oxidative stress and cardiovascular diseases-do anti-oxidative therapies fail?," Current Medicinal Chemistry, vol. 14, no. 16, pp. 17031712, 2007.

[8] A. Csordas, G. Wick, G. Laufer, and D. Bernhard, "An evaluation of the clinical evidence on the role of inflammation and oxidative stress in smoking-mediated cardiovascular disease," Biomarker Insights, vol. 3, pp. 127-139, 2008.

[9] A. Das, N. Dey, A. Ghosh, S. Das, D. J. Chattopadhyay, and I. B. Chatterjee, "Molecular and cellular mechanisms of cigarette smoke-induced myocardial injury: prevention by vitamin C," PloS One, vol. 7, no. 9, article e44151, 2012.

[10] J. Gvozdjak, A. Gvozdjáková, J. Kucharská, and V. Bada, “The effect of smoking on myocardial metabolism," Czechoslovak Medicine, vol. 10, no. 1, pp. 47-53, 1987.

[11] A. Gvozdjakova, V. Bada, L. Sány et al., "Smoke cardiomyopathy: disturbance of oxidative processes in myocardial mitochondria," Cardiovascular Research, vol. 18, no. 4, pp. 229-232, 1984.

[12] M. Alam, J. Wardell, E. Andersson, C. Höglund, and R. Nordlander, "Acute effects of smoking on diastolic function in healthy participants: studies by conventional doppler echocardiography and doppler tissue imaging," Journal of the American Society of Echocardiography, vol. 15, no. 10, Part 2, pp. 1232-1237, 2002.

[13] I. Barutcu, A. M. Esen, D. Kaya et al., "Effect of acute cigarette smoking on left and right ventricle filling parameters: a conventional and tissue Doppler echocardiographic study in healthy participants," Angiology, vol. 59, no. 3, pp. 312-316, 2008.

[14] O. Ciftci, M. Calişkan, H. Güllü, A. Yildirir, and H. Müderrisoğlu, "Mentholated cigarette smoking induced alterations in left and right ventricular functions in chronic smokers," Anadolu Kardiyoloji Dergisi, vol. 8, no. 2, pp. 116-122, 2008.

[15] E. Giacomin, E. Palmerini, P. Ballo, V. Zacà, G. Bova, and S. Mondillo, "Acute effects of caffeine and cigarette smoking on ventricular long-axis function in healthy subjects," Cardiovascular Ultrasound, vol. 6, p. 9, 2008.

[16] O. Gulel, K. Soylu, M. Yazici, S. Demircan, K. Durna, and M. Sahin, "Longitudinal diastolic myocardial functions are affected by chronic smoking in young healthy people: a study of color tissue Doppler imaging," Echocardiography, vol. 24, no. 5, pp. 494-498, 2007.

[17] T. F. Ilgenli and O. Akpinar, "Acute effects of smoking on right ventricular function. A tissue Doppler imaging study on healthy subjects," Swiss Medical Weekly, vol. 137, no. 5-6, pp. 91-96, 2007.

[18] O. Karakaya, I. Barutcu, A. M. Esen et al., "Acute smokinginduced alterations in Doppler echocardiographic measurements in chronic smokers," Texas Heart Institute Journal, vol. 33, no. 2, pp. 134-138, 2006.

[19] A. Leone, "Biochemical markers of cardiovascular damage from tobacco smoke," Current Pharmaceutical Design, vol. 11, no. 17, pp. 2199-2208, 2005.

[20] K. Narkiewicz, P. J. van de Borne, M. Hausberg et al., "Cigarette smoking increases sympathetic outflow in humans," Circulation, vol. 98, no. 6, pp. 528-534, 1998.

[21] H. M. Mertens, H. Mannebach, and U. Gleichmann, "Noninvasive effects of cigarette smoking on left ventricular function at rest and with exercise in normal individuals (author's transl)," Zeitschrift für Kardiologie, vol. 68, no. 6, pp. 374-381, 1979.

[22] M. A. Talukder, W. M. Johnson, S. Varadharaj et al., "Chronic cigarette smoking causes hypertension, increased oxidative stress, impaired NO bioavailability, endothelial dysfunction, and cardiac remodeling in mice," American Journal of Physiology. Heart and Circulatory Physiology, vol. 300, no. 1, pp. H388-H396, 2011.

[23] L. Gu, V. Pandey, D. L. Geenen, S. A. Chowdhury, and M. R. Piano, "Cigarette smoke-induced left ventricular remodelling is associated with activation of mitogen-activated protein kinases," European Journal of Heart Failure, vol. 10, no. 11, pp. 1057-1064, 2008.

[24] P. P. Santos, F. Oliveira, V. C. Ferreira et al., "The role of lipotoxicity in smoke cardiomyopathy," PloS One, vol. 9, no. 12, article e113739, 2014.

[25] D. R. Duarte, M. F. Minicucci, P. S. Azevedo et al., "The role of oxidative stress and lipid peroxidation in ventricular remodeling induced by tobacco smoke exposure after myocardial infarction," Clinics (São Paulo, Brazil), vol. 64, no. 7, pp. 691-7, 2009.

[26] A. Gvozdjakova, F. Simko, J. Kucharská, Z. Braunová, P. Psenek, and J. Kyselovic, "Captopril increased mitochondrial coenzyme Q10 level, improved respiratory chain function and energy production in the left ventricle in rabbits with smoke mitochondrial cardiomyopathy," BioFactors, vol. 10, no. 1, pp. 61-65, 1999.

[27] R. Novo, C. M. Freire, S. Felisbino et al., "Smoking is associated with remodeling of gap junction in the rat heart: smoker's paradox explanation?," Arquivos Brasileiros de Cardiologia, vol. 100, no. 3, pp. 274-280, 2013.

[28] D. R. Duarte, M. F. Minicucci, P. S. Azevedo et al., "Influence of lisinopril on cardiac remodeling induced by tobacco smoke exposure," Medical Science Monitor, vol. 16, no. 8, pp. BR255-BR259, 2010.

[29] J. M. Bradley, J. B. Nguyen, A. C. Fournett, and J. D. Gardner, "Cigarette smoke exacerbates ventricular remodeling and dysfunction in the volume overloaded heart," Microscopy and Microanalysis, vol. 18, no. 1, pp. 91-98, 2012.

[30] X. Zhou, G. An, and J. Chen, "Hydrogen sulfide improves left ventricular function in smoking rats via regulation of apoptosis and autophagy," Apoptosis, vol. 19, no. 6, pp. 998-1005, 2014.

[31] W. H. Herbert, "Cigarette smoking and arteriographically demonstrable coronary artery disease," Chest, vol. 67, no. 1, pp. 49-52, 1975.

[32] D. R. Ramsdale, E. B. Faragher, C. L. Bray, D. H. Bennett, C. Ward, and D. C. Beton, "Smoking and coronary artery disease assessed by routine coronary arteriography," British Medical Journal (Clinical Research Ed.), vol. 290, no. 6463, pp. 197-200, 1985.

[33] X. L. Wang, C. Tam, R. M. McCredie, and D. E. Wilcken, "Determinants of severity of coronary artery disease in Australian men and women," Circulation, vol. 89, no. 5, pp. 1974-1981, 1994.

[34] D. Waters, J. Lespérance, P. Gladstone et al., "Effects of cigarette smoking on the angiographic evolution of coronary atherosclerosis. A Canadian Coronary Atherosclerosis Intervention Trial (CCAIT) Substudy. CCAIT Study Group," Circulation, vol. 94, no. 4, pp. 614-621, 1996. 
[35] R. S. Barua, J. A. Ambrose, L. J. Eales-Reynolds, M. C. DeVoe, J. G. Zervas, and D. C. Saha, "Dysfunctional endothelial nitric oxide biosynthesis in healthy smokers with impaired endothelium-dependent vasodilatation," Circulation, vol. 104, no. 16, pp. 1905-1910, 2001.

[36] R. S. Barua, J. A. Ambrose, S. Srivastava, M. C. DeVoe, and L. J. Eales-Reynolds, "Reactive oxygen species are involved in smoking-induced dysfunction of nitric oxide biosynthesis and upregulation of endothelial nitric oxide synthase: an in vitro demonstration in human coronary artery endothelial cells," Circulation, vol. 107, no. 18, pp. 2342-2347, 2003.

[37] D. S. Celermajer, M. R. Adams, P. Clarkson et al., "Passive smoking and impaired endothelium-dependent arterial dilatation in healthy young adults," The New England Journal of Medicine, vol. 334, no. 3, pp. 150-154, 1996.

[38] D. S. Celermajer, K. E. Sorensen, D. Georgakopoulos et al., "Cigarette smoking is associated with dose-related and potentially reversible impairment of endotheliumdependent dilation in healthy young adults," Circulation, vol. 88, no. 5, Part 1, pp. 2149-2155, 1993.

[39] R. G. Ijzerman, E. H. Serne, M. M. van Weissenbruch, R. T. de Jongh, and C. D. Stehouwer, "Cigarette smoking is associated with an acute impairment of microvascular function in humans," Clinical Science (London, England), vol. 104, no. 3, pp. 247-252, 2003.

[40] W. G. Mayhan and G. M. Sharpe, "Effect of cigarette smoke extract on arteriolar dilatation in vivo," Journal of Applied Physiology (1985), vol. 81, no. 5, pp. 1996-2003, 1996.

[41] Y. Ota, K. Kugiyama, S. Sugiyama et al., "Impairment of endothelium-dependent relaxation of rabbit aortas by cigarette smoke extract-role of free radicals and attenuation by captopril," Atherosclerosis, vol. 131, no. 2, pp. 195202, 1997.

[42] H. Sumida, H. Watanabe, K. Kugiyama, M. Ohgushi, T. Matsumura, and H. Yasue, "Does passive smoking impair endothelium-dependent coronary artery dilation in women?," Journal of the American College of Cardiology, vol. 31, no. 4, pp. 811-815, 1998.

[43] W. Y. Craig, G. E. Palomaki, and J. E. Haddow, "Cigarette smoking and serum lipid and lipoprotein concentrations: an analysis of published data," $B M J$, vol. 298, no. 6676, pp. 784-788, 1989.

[44] B. Frei, T. M. Forte, B. N. Ames, and C. E. Cross, "Gas phase oxidants of cigarette smoke induce lipid peroxidation and changes in lipoprotein properties in human blood plasma. Protective effects of ascorbic acid," The Biochemical Journal, vol. 277, Part 1, pp. 133-138, 1991.

[45] T. Heitzer, S. Ylä-Herttuala, J. Luoma et al., "Cigarette smoking potentiates endothelial dysfunction of forearm resistance vessels in patients with hypercholesterolemia. Role of oxidized LDL," Circulation, vol. 93, no. 7, pp. 13461353, 1996.

[46] Y. Yamaguchi, S. Kagota, J. Haginaka, and M. Kunitomo, "Evidence of modified LDL in the plasma of hypercholesterolemic WHHL rabbits injected with aqueous extracts of cigarette smoke," Environmental Toxicology and Pharmacology, vol. 8, no. 4, pp. 255-260, 2000.

[47] E. A. Bermudez, N. Rifai, J. E. Buring, J. E. Manson, and P. M. Ridker, "Relation between markers of systemic vascular inflammation and smoking in women," The American Journal of Cardiology, vol. 89, no. 9, pp. 1117-9, 2002.
[48] C. J. Smith and T. H. Fischer, "Particulate and vapor phase constituents of cigarette mainstream smoke and risk of myocardial infarction," Atherosclerosis, vol. 158, no. 2, pp. 257267, 2001.

[49] P. S. Tappia, K. L. Troughton, S. C. Langley-Evans, and R. F. Grimble, "Cigarette smoking influences cytokine production and antioxidant defences," Clinical Science (London, England), vol. 88, no. 4, pp. 485-489, 1995.

[50] R. P. Tracy, B. M. Psaty, E. Macy et al., "Lifetime smoking exposure affects the association of $\mathrm{C}$-reactive protein with cardiovascular disease risk factors and subclinical disease in healthy elderly subjects," Arteriosclerosis, Thrombosis, and Vascular Biology, vol. 17, no. 10, pp. 2167-2176, 1997.

[51] D. Blache, "Involvement of hydrogen and lipid peroxides in acute tobacco smoking-induced platelet hyperactivity," The American Journal of Physiology, vol. 268, no. 2, Part 2, pp. H679-H685, 1995.

[52] Y. Fusegawa, S. Goto, S. Handa, T. Kawada, and Y. Ando, "Platelet spontaneous aggregation in platelet-rich plasma is increased in habitual smokers," Thrombosis Research, vol. 93, no. 6, pp. 271-278, 1999.

[53] J. Rival, J. M. Riddle, and P. D. Stein, "Effects of chronic smoking on platelet function," Thrombosis Research, vol. 45, no. 1, pp. 75-85, 1987.

[54] R. S. Barua, J. A. Ambrose, D. C. Saha, and L. J. Eales-Reynolds, "Smoking is associated with altered endothelial-derived fibrinolytic and antithrombotic factors: an in vitro demonstration," Circulation, vol. 106, no. 8, pp. 905-8, 2002.

[55] S. Matetzky, S. Tani, S. Kangavari et al., "Smoking increases tissue factor expression in atherosclerotic plaques: implications for plaque thrombogenicity," Circulation, vol. 102, no. 6, pp. 602-604, 2000.

[56] D. E. Newby, R. A. Wright, C. Labinjoh et al., "Endothelial dysfunction, impaired endogenous fibrinolysis, and cigarette smoking: a mechanism for arterial thrombosis and myocardial infarction," Circulation, vol. 99, no. 11, pp. 1411-1415, 1999.

[57] M. Pretorius, D. A. Rosenbaum, J. Lefebvre, D. E. Vaughan, and N. J. Brown, "Smoking impairs bradykininstimulated t-PA release," Hypertension, vol. 39, no. 3, pp. 767-771, 2002.

[58] T. P. Devasagayam, J. C. Tilak, K. K. Boloor, K. S. Sane, S. S. Ghaskadbi, and R. D. Lele, "Free radicals and antioxidants in human health: current status and future prospects," The Journal of the Association of Physicians of India, vol. 52, pp. 794-804, 2004.

[59] K. M. Holmstrom and T. Finkel, "Cellular mechanisms and physiological consequences of redox-dependent signalling," Nature Reviews. Molecular Cell Biology, vol. 15, no. 6, pp. 411-421, 2014.

[60] X. Zhou, C. Li, W. Xu, and J. Chen, "Trimetazidine protects against smoking-induced left ventricular remodeling via attenuating oxidative stress, apoptosis, and inflammation," PloS One, vol. 7, no. 7, article e40424, 2012.

[61] B. Bozkurt, S. B. Kribbs, F. J. Clubb Jr. et al., "Pathophysiologically relevant concentrations of tumor necrosis factor-alpha promote progressive left ventricular dysfunction and remodeling in rats," Circulation, vol. 97, no. 14, pp. 1382-1391, 1998.

[62] M. Bujak, M. Dobaczewski, K. Chatila et al., "Interleukin-1 receptor type I signaling critically regulates infarct healing 
and cardiac remodeling," The American Journal of Pathology, vol. 173, no. 1, pp. 57-67, 2008.

[63] D. Hasper, M. Hummel, F. X. Kleber, I. Reindl, and H. D. Volk, "Systemic inflammation in patients with heart failure," European Heart Journal, vol. 19, no. 5, pp. 761-5, 1998.

[64] M. Matsumoto, T. Tsujino, M. Lee-Kawabata et al., "Serum interleukin-6 and C-reactive protein are markedly elevated in acute decompensated heart failure patients with left ventricular systolic dysfunction," Cytokine, vol. 49, no. 3, pp. 264-268, 2010.

[65] K. Ono, A. Matsumori, T. Shioi, Y. Furukawa, and S. Sasayama, "Cytokine gene expression after myocardial infarction in rat hearts: possible implication in left ventricular remodeling," Circulation, vol. 98, no. 2, pp. 149-156, 1998.

[66] G. Torre-Amione, S. Kapadia, C. Benedict, H. Oral, J. B. Young, and D. L. Mann, "Proinflammatory cytokine levels in patients with depressed left ventricular ejection fraction: a report from the studies of left ventricular dysfunction (SOLVD)," Journal of the American College of Cardiology, vol. 27, no. 5, pp. 1201-1206, 1996.

[67] M. F. Huang, W. L. Lin, and Y. C. Ma, "A study of reactive oxygen species in mainstream of cigarette," Indoor Air, vol. 15, no. 2, pp. 135-140, 2005.

[68] A. L. Bluhm, J. Weinstein, and J. A. Sousa, "Free radicals in tobacco smoke,” Nature, vol. 229, no. 5285, p. 500, 1971.

[69] H. Witschi, "Carcinogenic activity of cigarette smoke gas phase and its modulation by beta-carotene and N-acetylcysteine," Toxicological Sciences, vol. 84, no. 1, pp. 81-87, 2005.

[70] K. F. Chung, "Inflammatory mediators in chronic obstructive pulmonary disease," Current Drug Targets. Inflammation and Allergy, vol. 4, no. 6, pp. 619-625, 2005.

[71] A. Churg, J. Dai, H. Tai, C. Xie, and J. L. Wright, “Tumor necrosis factor-alpha is central to acute cigarette smokeinduced inflammation and connective tissue breakdown," American Journal of Respiratory and Critical Care Medicine, vol. 166, no. 6, pp. 849-854, 2002.

[72] B. T. Mossman, K. M. Lounsbury, and S. P. Reddy, "Oxidants and signaling by mitogen-activated protein kinases in lung epithelium," American Journal of Respiratory Cell and Molecular Biology, vol. 34, no. 6, pp. 666-669, 2006.

[73] M. J. Walters, M. J. Paul-Clark, M. M. SK, K. Ito, I. M. Adcock, and J. A. Mitchell, "Cigarette smoke activates human monocytes by an oxidant-AP-1 signaling pathway: implications for steroid resistance," Molecular Pharmacology, vol. 68, no. 5, pp. 1343-1353, 2005.

[74] S. V. Culpitt, D. F. Rogers, P. Shah et al., "Impaired inhibition by dexamethasone of cytokine release by alveolar macrophages from patients with chronic obstructive pulmonary disease," American Journal of Respiratory and Critical Care Medicine, vol. 167, no. 1, pp. 24-31, 2003.

[75] R. Altara, M. Manca, R. Sabra, A. A. Eid, G. W. Booz, and F. A. Zouein, "Temporal cardiac remodeling post-myocardial infarction: dynamics and prognostic implications in personalized medicine," Heart Failure Reviews, 2015.

[76] X. Zhou, C. Li, W. Xu, and J. Chen, "Protective effects of valsartan against cigarette smoke-induced left ventricular systolic dysfunction in rats," International Journal of Cardio$\log y$, vol. 167, no. 3, pp. 677-680, 2013.

[77] Y. Arnson, Y. Shoenfeld, and H. Amital, "Effects of tobacco smoke on immunity, inflammation and autoimmunity," Journal of Autoimmunity, vol. 34, no. 3, pp. J258-J265, 2010.
[78] A. K. Khanna, J. Xu, and M. R. Mehra, “Antioxidant N-acetyl cysteine reverses cigarette smoke-induced myocardial infarction by inhibiting inflammation and oxidative stress in a rat model," Laboratory Investigation, vol. 92, no. 2, pp. 224235, 2012.

[79] P. P. dos Santos, B. F. Nogueira, B. P. Rafacho et al., "Aldosterone is not involved in the ventricular remodeling process induced by tobacco smoke exposure," Cellular Physiology and Biochemistry, vol. 30, no. 5, pp. 1191-1201, 2012.

[80] R. J. Mailloux, S. L. McBride, and M. E. Harper, "Unearthing the secrets of mitochondrial ROS and glutathione in bioenergetics," Trends in Biochemical Sciences, vol. 38, no. 12, pp. 592-602, 2013.

[81] I. Rahman and W. MacNee, "Antioxidant pharmacological therapies for COPD," Current Opinion in Pharmacology, vol. 12, no. 3, pp. 256-265, 2012.

[82] B. Aravamudan, M. A. Thompson, C. M. Pabelick, and Y. S. Prakash, "Mitochondria in lung diseases," Expert Review of Respiratory Medicine, vol. 7, no. 6, pp. 631-646, 2013.

[83] S. W. Ballinger, C. Patterson, C. N. Yan et al., "Hydrogen peroxide- and peroxynitrite-induced mitochondrial DNA damage and dysfunction in vascular endothelial and smooth muscle cells," Circulation Research, vol. 86, no. 9, pp. 960-966, 2000.

[84] C. A. Knight-Lozano, C. G. Young, D. L. Burow et al., "Cigarette smoke exposure and hypercholesterolemia increase mitochondrial damage in cardiovascular tissues," Circulation, vol. 105, no. 7, pp. 849-854, 2002.

[85] J. Lough, "Cardiomyopathy produced by cigarette smoke. Ultrastructural observations in guinea pigs," Archives of Pathology \& Laboratory Medicine, vol. 102, no. 7, pp. 377380, 1978.

[86] H. K. Thomsen and K. Kjeldsen, "Threshold limit for carbon monoxide-induced myocardial damage: an electron microscopic study in rabbits," Archives of Environmental Health, vol. 29, no. 2, pp. 73-78, 1974.

[87] T. S. Tippetts, D. R. Winden, A. C. Swensen et al., "Cigarette smoke increases cardiomyocyte ceramide accumulation and inhibits mitochondrial respiration," BMC Cardiovascular Disorders, vol. 14, p. 165, 2014.

[88] A. P. Wong, A. Niedzwiecki, and M. Rath, "Myocardial energetics and the role of micronutrients in heart failure: a critical review," American Journal of Cardiovascular Disease, vol. 6, no. 3, pp. 81-92, 2016.

[89] S. Yamada, X. Q. Zhang, T. Kadono et al., "Direct toxic effects of aqueous extract of cigarette smoke on cardiac myocytes at clinically relevant concentrations," Toxicology and Applied Pharmacology, vol. 236, no. 1, pp. 71-77, 2009.

[90] M. Minicucci, F. Oliveira, P. Santos et al., "Pentoxifylline attenuates cardiac remodeling induced by tobacco smoke exposure," Arquivos Brasileiros de Cardiologia, vol. 106, no. 5, pp. 396-403, 2016.

[91] G. Pearson, F. Robinson, T. Beers Gibson et al., "Mitogenactivated protein (MAP) kinase pathways: regulation and physiological functions," Endocrine Reviews, vol. 22, no. 2, pp. 153-183, 2001.

[92] J. R. Testa and P. N. Tsichlis, "AKT signaling in normal and malignant cells," Oncogene, vol. 24, no. 50, pp. 73917393, 2005.

[93] X. Zhou, C. Li, and X. Lu, "The pathogenic role of autophagy in smoking-induced left ventricular systolic dysfunction in 
rats," International Journal of Cardiology, vol. 168, no. 4, pp. 4302-4303, 2013.

[94] J. W. Stephens and S. E. Humphries, "The molecular genetics of cardiovascular disease: clinical implications," Journal of Internal Medicine, vol. 253, no. 2, pp. 120-127, 2003.

[95] P. J. Talmud, J. W. Stephens, E. Hawe et al., "The significant increase in cardiovascular disease risk in APOEepsilon4 carriers is evident only in men who smoke: potential relationship between reduced antioxidant status and ApoE4," Annals of Human Genetics, vol. 69, Part 6, pp. 613-622, 2005.

[96] Y. Chen, P. T. Dawes, J. C. Packham, and D. L. Mattey, "Interaction between smoking and functional polymorphism in the TGFB1 gene is associated with ischaemic heart disease and myocardial infarction in patients with rheumatoid arthritis: a cross-sectional study," Arthritis Research \& Therapy, vol. 14, no. 2, p. R81, 2012.

[97] P. de Moerloose and F. Boehlen, "Inherited thrombophilia in arterial disease: a selective review," Seminars in Hematology, vol. 44, no. 2, pp. 106-113, 2007.

[98] M. L. Grisoni, C. Proust, M. Alanne; et al., "Haplotypic analysis of tag SNPs of the interleukin-18 gene in relation to cardiovascular disease events: the MORGAM project," European Journal of Human Genetics, vol. 16, no. 12, pp. 1512-1520, 2008.

[99] L. P. Breitling, R. Yang, B. Korn, B. Burwinkel, and H. Brenner, "Tobacco-smoking-related differential DNA methylation: 27K discovery and replication," American Journal of Human Genetics, vol. 88, no. 4, pp. 450-457, 2011.

[100] E. S. Wan, W. Qiu, A. Baccarelli et al., "Cigarette smoking behaviors and time since quitting are associated with differential DNA methylation across the human genome," Human Molecular Genetics, vol. 21, no. 13, pp. 3073-3082, 2012.

[101] L. P. Breitling, K. Salzmann, D. Rothenbacher, B. Burwinkel, and H. Brenner, "Smoking, F2RL3 methylation, and prognosis in stable coronary heart disease," European Heart Journal, vol. 33, no. 22, pp. 2841-2848, 2012.

[102] J. J. McDougall, C. Zhang, L. Cellars, E. Joubert, C. M. Dixon, and N. Vergnolle, "Triggering of proteinase-activated receptor 4 leads to joint pain and inflammation in mice," Arthritis and Rheumatism, vol. 60, no. 3, pp. 728-737, 2009.

[103] J. D. Muehlschlegel, T. E. Perry, K. Y. Liu et al., "Polymorphism in the protease-activated receptor-4 gene region associates with platelet activation and perioperative myocardial injury," American Journal of Hematology, vol. 87, no. 2, pp. 161-166, 2012.

[104] C. C. Wu, S. Y. Wu, C. Y. Liao, C. M. Teng, Y. C. Wu, and S. C. Kuo, "The roles and mechanisms of PAR4 and P2Y12/phosphatidylinositol 3-kinase pathway in maintaining thrombin-induced platelet aggregation," British Journal of Pharmacology, vol. 161, no. 3, pp. 643-658, 2010.

[105] E. L. Marczylo, A. A. Amoako, J. C. Konje, T. W. Gant, and T. H. Marczylo, "Smoking induces differential miRNA expression in human spermatozoa: a potential transgenerational epigenetic concern?," Epigenetics, vol. 7, no. 5, pp. 432-439, 2012.

[106] M. J. Maenner, L. C. Denlinger, A. Langton, K. J. Meyers, C. D. Engelman, and H. G. Skinner, "Detecting gene-bysmoking interactions in a genome-wide association study of early-onset coronary heart disease using random forests," BMC Proceedings, vol. 3, Supplement 7, p. S88, 2009.
[107] S. F. Ali, E. E. Smith, D. L. Bhatt, G. C. Fonarow, and L. H. Schwamm, "Paradoxical association of smoking with inhospital mortality among patients admitted with acute ischemic stroke," Journal of the American Heart Association, vol. 2, no. 3, article e000171, 2013.

[108] G. I. Barbash, J. Reiner, H. D. White et al., "Evaluation of paradoxic beneficial effects of smoking in patients receiving thrombolytic therapy for acute myocardial infarction: mechanism of the "smoker's paradox" from the GUSTO-I trial, with angiographic insights. Global Utilization of Streptokinase and Tissue-Plasminogen Activator for Occluded Coronary Arteries," Journal of the American College of Cardiology, vol. 26, no. 5, pp. 1222-1229, 1995.

[109] A. H. Kitchin and S. J. Pocock, "Prognosis of patients with acute myocardial infarction admitted to a coronary care unit. I: survival in hospital," British Heart Journal, vol. 39, no. 11, pp. 1163-1166, 1977.

[110] J. S. Pollock, R. D. Hollenbeck, L. Wang, D. R. Janz, T. W. Rice, and J. A. McPherson, "A history of smoking is associated with improved survival in patients treated with mild therapeutic hypothermia following cardiac arrest," Resuscitation, vol. 85, no. 1, pp. 99-103, 2014.

[111] T. Gupta, D. Kolte, S. Khera et al., "Smoker's paradox in patients with ST-segment elevation myocardial infarction undergoing primary percutaneous coronary intervention," Journal of the American Heart Association, vol. 5, no. 4, 2016.

[112] S. A. Paiva, R. Novo, B. B. Matsubara et al., "Beta-carotene attenuates the paradoxical effect of tobacco smoke on the mortality of rats after experimental myocardial infarction," The Journal of Nutrition, vol. 135, no. 9, pp. 2109-2113, 2005.

[113] Y. J. Zhang, J. Iqbal, D. van Klaveren et al., "Smoking is associated with adverse clinical outcomes in patients undergoing revascularization with PCI or CABG: the SYNTAX trial at 5-year follow-up," Journal of the American College of Cardiology, vol. 65, no. 11, pp. 1107-1115, 2015.

[114] B. Acar, O. Ozeke, S. Unal et al., "Change in left ventricular systolic function in patients with ST elevation myocardial infarction: evidence for smoker's paradox or pseudo-paradox?," Indian Heart Journal, vol. 68, no. 6, pp. 816-820, 2016.

[115] G. Gennaro, S. J. Brener, B. Redfors et al., "Effect of smoking on infarct size and major adverse cardiac events in patients with large anterior ST-elevation myocardial infarction (from the INFUSE-AMI trial)," The American Journal of Cardiology, vol. 118, no. 8, pp. 1097-1104, 2016.

[116] M. Saad, G. Fuernau, S. Desch et al., “'Smoker's paradox” in patients with cardiogenic shock complicating myocardial infarction - a substudy of the IABP-SHOCK II-trial and registry," International Journal of Cardiology, vol. 222, pp. 775779, 2016.

[117] M. P. Ryan and J. J. Hinojosa, "Conceptual obstacles to making use of four smoking-cessation strategies: what reasons do light smokers give for rejecting strategies?," Health Psychology Open, vol. 2, no. 2, article 2055102915624928, 2015.

[118] L. F. Stead, R. Perera, C. Bullen et al., "Nicotine replacement therapy for smoking cessation," Cochrane Database of Systematic Reviews, vol. 11, article CD000146, 2012.

[119] P. M. Cinciripini, D. W. Wetter, and J. B. McClure, "Scheduled reduced smoking: effects on smoking abstinence and potential mechanisms of action," Addictive Behaviors, vol. 22, no. 6, pp. 759-767, 1997.

[120] D. Shapiro, G. E. Schwartz, B. Tursky, and S. R. Shnidman, "Smoking on cue: a behavioral approach to smoking 
reduction," Journal of Health and Social Behavior, vol. 12, no. 2, pp. 108-113, 1971.

[121] K. Morphett, B. Partridge, C. Gartner, A. Carter, and W. Hall, "Why don't smokers want help to quit? A qualitative study of smokers' attitudes towards assisted vs. unassisted quitting," International Journal of Environmental Research and Public Health, vol. 12, no. 6, pp. 6591-6607, 2015.

[122] A. Black, E. Beard, J. Brown, J. Fidler, and R. West, "Beliefs about the harms of long-term use of nicotine replacement therapy: perceptions of smokers in England," Addiction, vol. 107, no. 11, pp. 2037-2042, 2012.

[123] B. P. Rafacho, P. Santos, H. B. Assalin et al., "Role of vitamin $\mathrm{D}$ in the cardiac remodeling induced by tobacco smoke exposure," International Journal of Cardiology, vol. 155, no. 3, pp. 472-3, 2012.

[124] S. I. Bibli, I. Andreadou, C. Glynos et al., "Exposure to cigarette smoke abrogates the beneficial effect of ischemic postconditioning," American Journal of Physiology. Heart and Circulatory Physiology, vol. 311, no. 5, pp. H1321H1332, 2016.

[125] X. Zhou, L. Zhao, J. Mao, J. Huang, and J. Chen, “Antioxidant effects of hydrogen sulfide on left ventricular remodeling in smoking rats are mediated via PI3K/Akt-dependent activation of Nrf2," Toxicological Sciences, vol. 144, no. 1, pp. 197-203, 2015.

[126] D. Reis Junior, E. L. Antonio, M. F. de Franco, H. A. de Oliveira, P. J. Tucci, and A. J. Serra, "Association of exercise training with tobacco smoking prevents fibrosis but has adverse impact on myocardial mechanics," Nicotine \& Tobacco Research, vol. 18, no. 12, pp. 2268-2272, 2016.

[127] L. A. Zornoff, D. R. Duarte, M. F. Minicucci et al., "Effects of beta-carotene and smoking on heart remodeling after myocardial infarction," Arquivos Brasileiros de Cardiologia, vol. 89, no. 3, pp. 135-141, 2007, 151-7.

[128] D. R. Duarte, L. C. Oliveira, M. F. Minicucci et al., "Effects of the administration of beta-blockers on ventricular remodeling induced by cigarette smoking in rats," Arquivos Brasileiros de Cardiologia, vol. 92, no. 6, pp. 443-447, 2009, 462$6,479-83$.

[129] F. Denipote, L. P. Ardisson, P. S. Azevedo et al., "Influence of taurine on cardiac remodeling induced by tobacco smoke exposure," Cellular Physiology and Biochemistry, vol. 27, no. 3-4, pp. 291-298, 2011.

[130] B. Zhu, Y. Sun, R. E. Sievers et al., "L-arginine decreases infarct size in rats exposed to environmental tobacco smoke," American Heart Journal, vol. 132, no. 1, Part 1, pp. 91-100, 1996. 


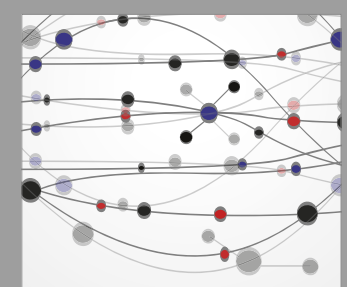

The Scientific World Journal
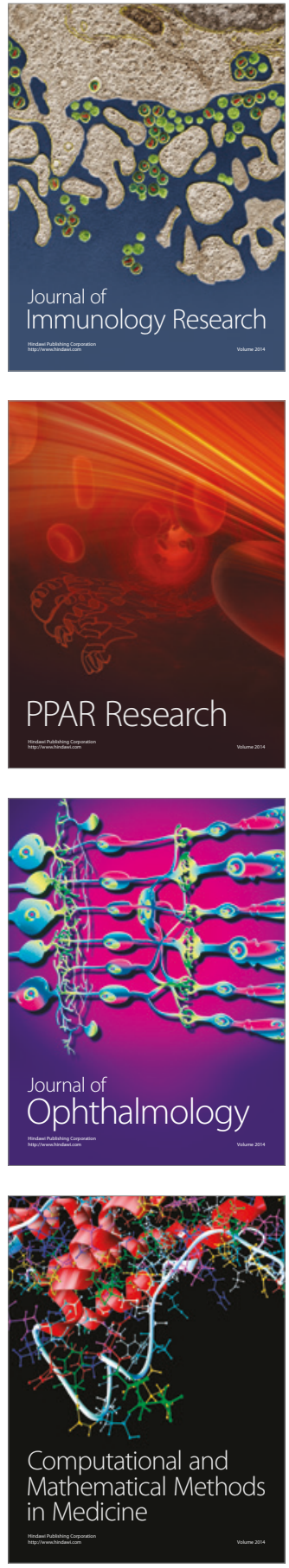

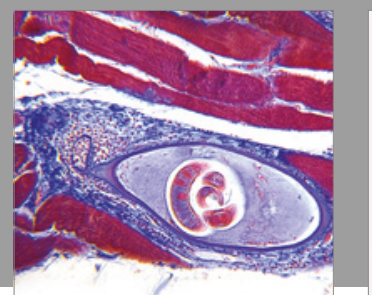

Gastroenterology Research and Practice
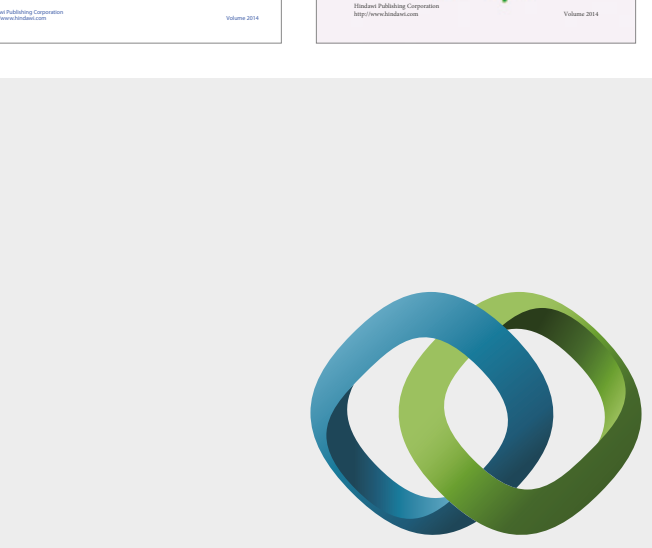

\section{Hindawi}

Submit your manuscripts at

https://www.hindawi.com
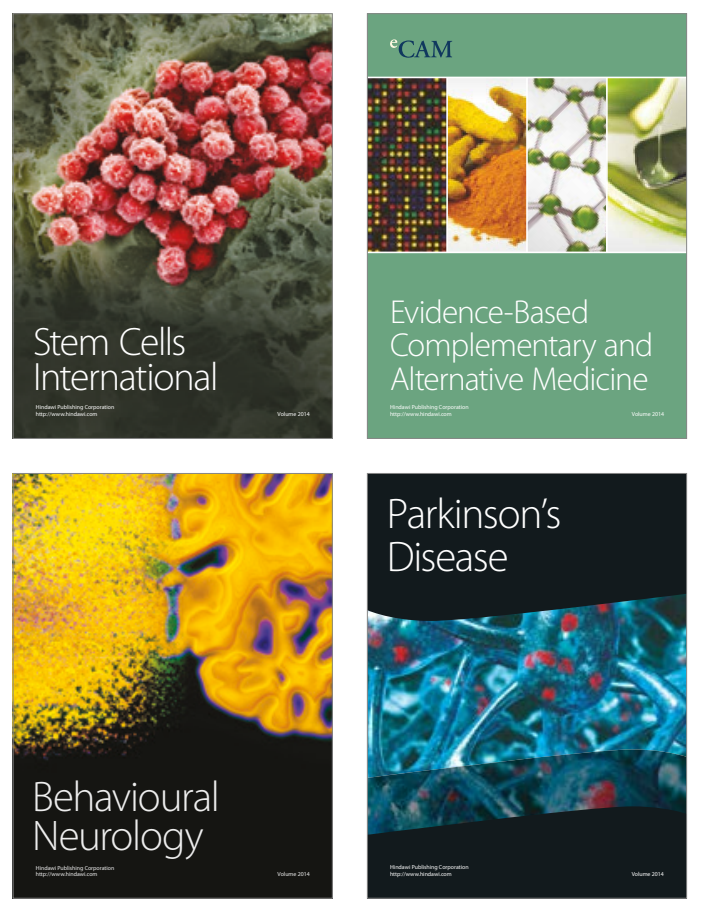
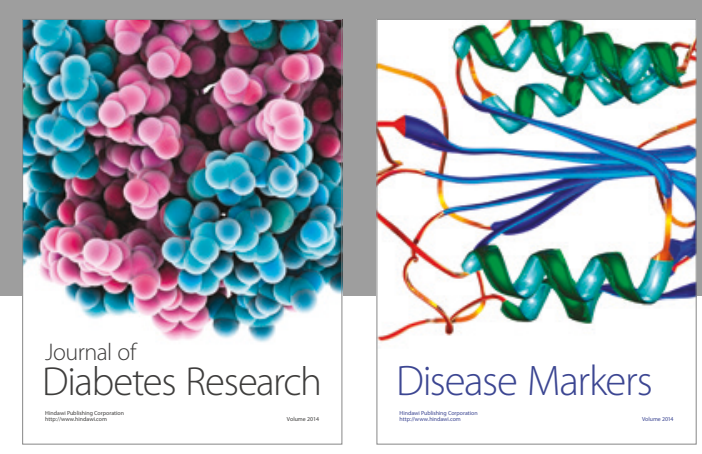

Disease Markers
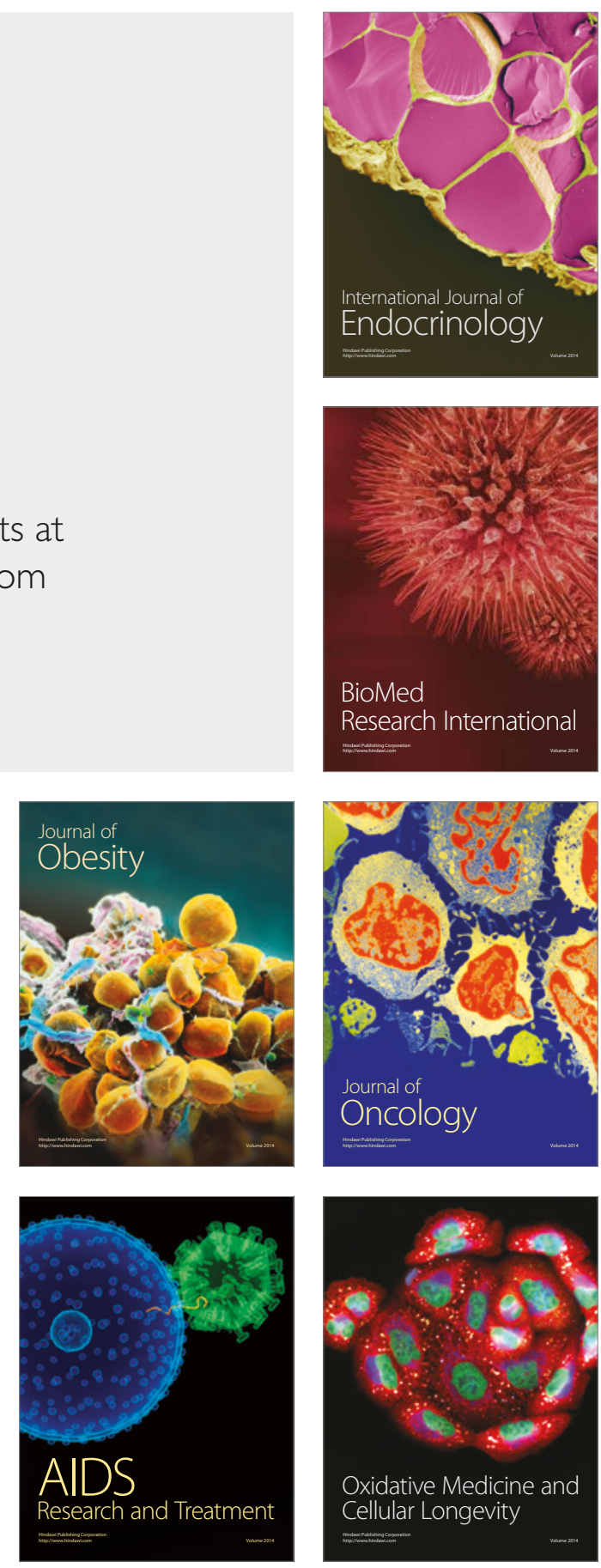